\title{
Fermi-Bose Similarity, Supersymmetry and Generalized Numbers.
}

\author{
Y. OHNUKI
}

Department of Physics, Nagoya University - Nagoya 464, Japan

S. KAMEFUCHI

Institute of Physics, Tsukuba University - Ibaraki 305, Japan

(Nuovo Cimento $A, \mathbf{7 0}, 435(1982))$

p. 445: The left-hand side of (4.3) should read $\tilde{x}^{|x|}$.

p. 448: The first term within the large parentheses on the right-hand side of (4.19) should also contain the factor $\left(1+\delta_{s s^{\prime}}\right)$.

p. 449: The subscript $\{j\}$ attached to $\Sigma$ and $G$ appearing on the right-hand side of (4.21) should be replaced by $\left\{j^{\prime}\right\}$; the equation in the 3rd line from (4.22) should read

$$
\left|\varrho_{j_{a}}^{(a)}\right|=\left|\varrho_{j_{a}^{\prime}}^{(a) \prime}\right|
$$

p. 452: The reference to be quoted in the 3 rd line is $\left({ }^{9}\right)$, and the footnote thereof should be shifted to p. 459 .

p. 454: In the 7th line $(\overline{i j})$ should be replaced by $(i j)$.

p. 457: The $x_{i}$ 's appearing in the 2 nd equation in (7.2) should both be replaced by $\hat{x}_{i}$; the last subscript among those attarhed to $\Sigma$ on the right-hand side of (7.5) should read $r_{n}$.

(C) by Società Italiana di Fisica

Proprieta letteraria riservata

Direttore responsabile: RENATO ANGELO RICCI

Stampato in Bologna dalla Tipografia Compositori coi tipi della Tipografia Monograf
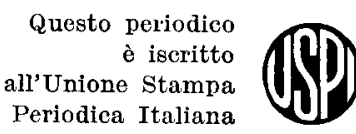

Questo fascicolo è stato licenziato dai torohi il 15-II-1983 